11

\title{
Электрическое поле горизонтальной линейной заводненной антенны
}

\author{
() Е.Д. Терещенко, ${ }^{1}$ П.Е. Терещенко ${ }^{2}$ \\ ${ }^{1}$ Полярный геофизический институт, \\ 183010 Мурманск, Россия \\ ${ }^{2}$ Институт земного магнетизма, ионосферы и распространения радиоволн им. Н.В. Пушкова РАН, \\ 199034 Санкт-Петербург, Россия \\ e-mail: tereshchenko@gmail.com
}

(Поступило в редакцию 7 июня 2016 г. В окончательной редакции 8 сентября 2016 г.)

Рассмотрено возбуждение электрического поля горизонтальным заводненным источником, находящимся на границе раздела двух сред. Решение задачи представлено в виде интегралов, содержащих быстроосциллирующую функцию Бесселя. В рамках квазистационарного приближения общие формулы, описывающие поле в воде, представлены с помощью интегралов Ватсона через хорошо изученные модифицированные функции Бесселя. Показано, что в зоне, находящейся на расстоянии больше скин-слоя от антенны, вертикальная компонента определяется полем, распространяющимся исключительно в нижней среде, а компоненты, перпендикулярные антенне, имеют вид волн, распространяющихся в верхней среде без поглощения, впоследствии проникающих вглубь, изменяясь по экспоненциальному закону.

DOI: 10.21883/JTF.2017.03.44254.1917

\section{Введение}

Задача возбуждения электромагнитных волн источником, находящимся на границе раздела двух сред, имеет богатую историю, начиная с работ Зоммерфельда [1], Вейля [2], Фока, Бурсиана [3], а также более близких к настоящему времени работ Уэйта [4], Макарова [5], Вешева [6], Кинга [7] и др. Однако остаются некоторые вопросы, не нашедшие своего освещения в известной литературе, и в связи с развитием систем управления глубоко погруженными объектами, а также электромагнитных методов исследования литосферы океана [8], вновь возникает интерес к подобным задачам. В отличие от экспериментов на земной поверхности, где измеряемой величиной является поле на границе раздела, при проведении морских работ немаловажную роль играет информация об изменении поля с глубиной.

В настоящей работе получим аналитические формулы, описывающие структуру электрического поля, возбуждаемого горизонтальной линейной антенной с заводненными электродами, которую для краткости будем называть „заводненной“, и его изменение с глубиной. В рамках квазистационарного метода, являющегося хорошим приближением при описании волн крайне низкочастотного (КНЧ) и более низкого диапазонов, представим компоненты поля в виде хорошо известных функций. В отличие от широко распространенного подхода к вычислению поля на границе раздела с использованием интегралов Фока $[3,6]$ воспользуемся двумя интегралами Ватсона [9], позволяющими определить не только поле на границе раздела, но и его изменение с глубиной.

\section{1. Электрический вектор-потенциал и его связь с электрическим полем}

Рассмотрим излучение в двуслойной среде заводненной антенны длиной $2 L$, питаемой током с гармони- ческой зависимостью от времени $\exp (-i \omega t)$. Систему координат выбираем следующим (см. рисунок) образом. Центр декартовых координат помещаем в середину антенны, ось $z$ направляем вверх, ось $x$ - вдоль антенны, ось $y$ - поперек антенны. Расстояние до точки наблюдения обозначим $R$, а расстояние на плоскости $(x, y, 0)$ обозначим $\rho$. Среду в области $z>0$ считаем практически непроводящей $(\sigma=+0$, наличие + у нуля указывает на небольшое поглощение) с диэлектрической проницаемостью $\epsilon_{0} \approx\left(10^{-9}\right) /(36 \pi) \mathrm{F} / \mathrm{m}$ и магнитной проницаемостью $\mu_{0}=4 \pi \cdot 10^{-7} \mathrm{H} / \mathrm{m}$. Предполагаем, что область $z<0$ имеет электромагнитные параметры $\epsilon_{1}, \mu_{0}, \sigma_{1}$.

Задача возбуждения электромагнитного поля сторонним током $\mathbf{J}$ сводится к решению уравнений Гельмгольца для электрического вектора-потенциала А с соответствующими граничными условиями $[4,6]$. Рассматриваем излучение монохроматических волн, поэтому будем использовать уравнения для комплексных амплитуд $\mathbf{A}$, соответствующих монохроматических компонент $(\mathbf{A} \rightarrow \mathbf{A} \exp (-i \omega t), \mathbf{E} \rightarrow \mathbf{E} \exp (-i \omega t), \mathbf{E}$-электрическое поле).

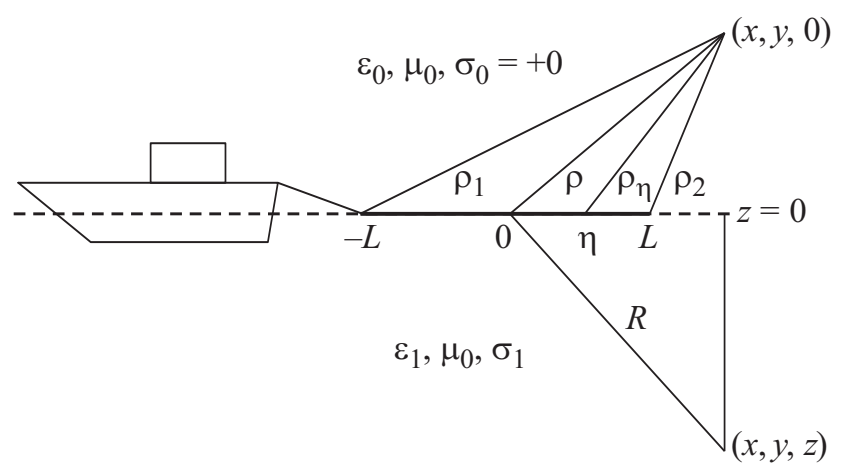

Геометрия задачи. 
Принимая во внимание, что источник направлен вдоль оси $x$ (см. рисунок), представляем вектор А в следующем виде:

$$
\mathbf{A}^{(j)}=A_{x}^{(j)} \mathbf{e}_{x}+A_{z}^{(j)} \mathbf{e}_{z},
$$

где $j=0,1$ указывает на среду, $\mathbf{e}_{x}$ и $\mathbf{e}_{z}-$ единичные орты, направленные вдоль осей $x$ и z соответственно.

Дальнейшая задача сводится к нахождению решения системы уравнений

$$
\nabla^{2} \mathbf{A}^{(j)}+k_{j}^{2} \mathbf{A}^{(j)}=-\mathbf{J}, \quad j=0,1
$$

с граничными условиями

$$
\begin{gathered}
\left.\mathbf{A}^{(0)}\right|_{z=0}=\left.\mathbf{A}^{(1)}\right|_{z=0},\left.\quad \frac{\partial A_{x}^{(0)}}{\partial z}\right|_{z=0}=\left.\frac{\partial A_{x}^{(1)}}{\partial z}\right|_{z=0}, \\
\left.\frac{1}{k_{0}^{2}} \operatorname{div} \mathbf{A}^{(0)}\right|_{z=0}=\left.\frac{1}{k_{1}^{2}} \operatorname{div} \mathbf{A}^{(1)}\right|_{z=0} .
\end{gathered}
$$

Волновые числа $k_{0}$ и $k_{1}$ входящие в систему уравнений (2) и (3), определяются выражениями

$$
\begin{gathered}
k_{0}=\frac{\omega}{c}(1+i 0)=\frac{\omega}{c}, \\
k_{1}=\frac{\omega}{c} \sqrt{\frac{\varepsilon_{1}}{\varepsilon_{0}}+i \frac{\sigma_{1}}{\omega \varepsilon_{0}}}=\frac{\omega}{c} \sqrt{\tilde{\varepsilon}_{1}^{\prime}} .
\end{gathered}
$$

Рассмотрим решение системы (2) с граничными условиями (3) для точечного заводненного горизонтального источника, расположенного в начале координат. В этом случае

$$
\begin{aligned}
& \mathbf{J}^{(0)}=J \Delta_{x} \delta(x) \delta(y) \delta(z-0) \mathbf{e}_{x}, \\
& \mathbf{J}^{(1)}=J \Delta_{x} \delta(x) \delta(y) \delta(z+0) \mathbf{e}_{x},
\end{aligned}
$$

где $\delta$ - дельта-функция, $J-$ ток, $J \Delta_{x}-$ дипольный момент, $\Delta_{x}$ - длина диполя, стремящаяся к бесконечно малой величине.

Переход к величинам, соответствующим возбуждению линейной антенной длиной $2 L$, осуществляется с помощью суммирования по длине антенны. В частности, $\boldsymbol{\varepsilon}-$ электрическое поле, возбуждаемое линейной антенной, будет равно

$$
\boldsymbol{\varepsilon}^{(j)}=\Sigma \mathbf{E}^{(j)}\left(\rho_{\eta}, z\right),
$$

где $\mathbf{E}^{(j)}\left(\rho_{\eta}, z\right)$ - поле диполя, находящегося в точке $\eta$ антенны.

Решение системы (2) с граничными условиями (3) строится в цилиндрической системе координат $(z, \rho, \phi)$ в виде разложения по базисным функциям $\cos \frac{m}{2} \phi$, $m=0,1, \ldots$, образующим полную систему на промежутке $(0,2 \pi][9]$.

Дальнейшие шаги хорошо известны $[5,6]$ и связаны с определением с учетом граничных условий соответствующих функций, входящих в разложение и зависящих от $\rho, z$. Поэтому, опуская довольно несложные преобразования, приведем конечный результат вычисления $A_{x}^{(j)}$ и $A_{z}^{(j)}$ для источника (5)

$$
A_{x}^{(j)}=\frac{J \cdot \Delta_{x}}{4 \pi} \int_{0}^{\infty} \frac{2 i \exp \left(i \sqrt{k_{j}^{2}-\lambda^{2}}|z|\right)}{\sqrt{k_{0}^{2}-\lambda^{2}}+\sqrt{k_{1}^{2}-\lambda^{2}}} J_{0}(\lambda \rho) \lambda d \lambda,
$$

$$
\begin{aligned}
& A_{z}^{(j)}=\frac{J \cdot \Delta_{x}}{4 \pi} \frac{\partial}{\partial x}\left(k_{0}^{2}-k_{1}^{2}\right) \\
& \times \int_{0}^{\infty} \frac{2 \exp \left(i \sqrt{k_{j}^{2}-\lambda^{2}}|z|\right)}{\left(\sqrt{k_{0}^{2}-\lambda^{2}}+\sqrt{\left.k_{1}^{2}-\lambda^{2}\right)\left(k_{1}^{2} \sqrt{k_{0}^{2}-\lambda^{2}}+k_{0}^{2} \sqrt{k_{1}^{2}-\lambda^{2}}\right)}\right.} \\
& \times J_{0}(\lambda \rho) \lambda d \lambda,
\end{aligned}
$$

$j=0.1$. Ветвь квадратного корня фиксировали, исходя из условия

$$
\operatorname{Im} \sqrt{k_{j}^{2}-\lambda^{2}}>0 .
$$

Для компактности записи, а также чтобы иметь аналогию с работами по геоэлектрике $[3,6]$, преобразуем формулу (7), введя новые обозначения

$$
\begin{gathered}
k_{j}=i \varkappa_{j}, \quad v_{j}=-i \sqrt{k_{j}^{2}-\lambda^{2}}=\sqrt{\varkappa_{j}^{2}+\lambda^{2}}, \\
A_{x}^{(j)}=\frac{j \cdot \Delta_{x}}{4 \pi} \Pi^{(j)}(\rho, z), \quad A_{z}^{(j)}=\frac{J \cdot \Delta_{x}}{4 \pi} \frac{\partial}{\partial x} F^{(j)}(\rho, z) .
\end{gathered}
$$

Из соотношения (8) следует $\operatorname{Re} v_{j}>0$, а из (9) и (7) получаем

$$
\begin{gathered}
\Pi^{(j)}(\rho, z)=\int_{0}^{\infty} \frac{2}{v_{0}+v_{1}} \exp \left(-v_{j}|z|\right) J_{0}(\lambda \rho) \lambda d \lambda, \\
F^{(j)}(\rho, z)=\left(\varkappa_{1}^{2}-\varkappa_{0}^{2}\right) \\
\times \int_{0}^{\infty} \frac{2}{\left(v_{0}+v_{1}\right)\left(\varkappa_{0}^{2} v_{1}+\varkappa_{1}^{2} v_{0}\right)} \exp \left(-v_{j}|z|\right) J_{0}(\lambda \rho) \lambda d \lambda, \\
j=0,1 .
\end{gathered}
$$

Система (10) является базовой при вычислении электрического поля. Рассмотрим практически важный случай - поле в нижней среде (в воде). Принимая во внимание, что электрическое поле $\mathbf{E}^{(j)}$ связано с электрическим вектор-потенциалом следующим соотношением:

$$
\mathbf{E}^{(1)}=i \omega \mu_{0} \mathbf{A}^{(1)}-\operatorname{grad} \frac{\operatorname{div} \mathbf{A}^{(1)}}{i \omega \tilde{\varepsilon}_{1}^{\prime} \varepsilon_{0}},
$$

a

$$
\mathbf{A}^{(1)}=A_{x}^{(1)} \mathbf{e}_{x}+A_{z}^{(1)} \mathbf{e}_{z},
$$

получим с учетом (9)

$$
\begin{gathered}
E_{x}^{(1)}=\frac{i \omega \mu_{0}}{4 \pi} J \cdot \Delta_{x}\left[\Pi^{(1)}(\rho, z)-\frac{\partial}{\partial x} \frac{1}{\varkappa_{1}^{2}} \frac{\partial}{\partial x} P^{(1)}(\rho, z)\right] \\
E_{y}^{(1)}=-\frac{i \omega \mu_{0}}{4 \pi} J \cdot \Delta_{x} \frac{\partial}{\partial x}\left[\frac{1}{\varkappa_{1}^{2}} \frac{\partial}{\partial y} P^{(1)}(\rho, z)\right] \\
E_{z}^{(1)}=\frac{i \omega \mu_{0}}{4 \pi} J \cdot \Delta_{x} \frac{\partial}{\partial x} S^{(1)}(\rho, z)
\end{gathered}
$$

где обозначено

$$
P^{(1)}(\rho, z)=\Pi^{(1)}(\rho, z)+\frac{\partial}{\partial z} F^{(1)}(\rho, z),
$$




$$
S^{(1)}(\rho, z)=F^{(1)}(\rho, z)-\frac{1}{\chi_{1}^{2}} \frac{\partial}{\partial z} P^{(1)}(\rho, z) .
$$

Воспользуемся (10), тогда можно будет представить функции, определяющие $\mathbf{E}^{(1)}$, через интегралы, содержащие функции Бесселя:

$$
\begin{gathered}
\Pi^{(1)}(\rho, z)=\int_{0}^{\infty} \frac{2}{v_{0}+v_{1}} \exp \left(v_{1} z\right) J_{0}(\lambda \rho) \lambda d \lambda, \\
P^{(1)}(\rho, z)=\varkappa_{1}^{2} \int_{0}^{\infty} \frac{2}{x_{0}^{2} \nu_{1}+\varkappa_{1}^{2} \nu_{0}} \exp \left(v_{1} z\right) J_{0}(\lambda \rho) \lambda d \lambda, \\
S^{(1)}(\rho, z)=-\int_{0}^{\infty} \frac{2 v_{0}}{x_{0}^{2} \nu_{1}+\varkappa_{1}^{2} \nu_{0}} \exp \left(v_{1} z\right) J_{0}(\lambda \rho) \lambda d \lambda .
\end{gathered}
$$

Таким образом, имеем несобственные интегралы, содержащие осциллирующую функцию $J_{0}(\lambda \rho)$. Для вычисления таких интегралов существуют хорошо разработанные численные методы, в частности метод Лонгмана $[10,11]$. Для практически важного случая КНЧ и более низкого диапазона формулы (14) допускают упрощение и возможность их представления через хорошо исследованные функции.

\section{2. Квазистационарное приближение}

В квазистационарном приближении полагают $\varkappa_{0}=0$. Физически это соответствует пренебрежению максвелловским током смещения по сравнению с током проводимости. При использовании волн очень низкой частоты хорошо выполняется условие $\left|\varkappa_{0}^{2} / \varkappa_{1}^{2}\right| \ll 1$, что дает возможность заменить в формулах (14) $\varkappa_{0}$ нулем, а $v_{0} \rightarrow \lambda$. Тогда будем иметь

$$
\begin{gathered}
\Pi(\rho, z)=\left.\Pi^{(1)}(\rho, z)\right|_{\varkappa_{0}=0}=\int_{0}^{\infty} \frac{2 \exp \left(v_{1} z\right)}{v_{1}+\lambda} J_{0}(\lambda \rho) \lambda d \lambda, \\
P(\rho, z)=\left.P^{(1)}(\rho, z)\right|_{\varkappa_{0}=0}=\int_{0}^{\infty} 2 \exp \left(v_{1} z\right) J_{0}(\lambda \rho) d \lambda, \\
S(\rho, z)=\left.S^{(1)}(\rho, z)\right|_{\varkappa_{0}=0}=-\frac{1}{\varkappa_{1}^{2}} \int_{0}^{\infty} 2 \exp \left(v_{1} z\right) J_{0}(\lambda \rho) \lambda d \lambda .
\end{gathered}
$$

Интегралы, определяющие П $(\rho, z), P(\rho, z)$ и $S(\rho, z)$, являются производными от двух интегралов Ватсона: один, определяющий падающее поле [5]

$$
\begin{gathered}
\int_{0}^{\infty} \frac{\exp \left(v_{1} z\right)}{v_{1}} J_{0}(\lambda \rho) \lambda d \lambda=\frac{\exp \left(-\varkappa_{1} R\right)}{R}=\frac{\exp \left(i k_{1} R\right)}{R} \\
R=\sqrt{\rho^{2}+z^{2}}
\end{gathered}
$$

другой связан с произведением модифицированных функций Бесселя [9]

$$
\int_{0}^{\infty} \frac{\exp \left(v_{1} z\right)}{v_{1}} J_{0}(\lambda \rho) d \lambda=I_{0}\left(r_{+}\right) K_{0}\left(r_{-}\right),
$$

где

$$
r_{+}=\frac{\varkappa_{1}}{2}(R+z), \quad r_{-}=\frac{\varkappa_{1}}{2}(R-z),
$$

а $I_{0}$ и $K_{0}$ - модифицированные функции Бесселя.

Вычислим функции П $(\rho, z), P(\rho, z)$ и $S(\rho, z)$, используя интегралы Ватсона (16) и (17):

$$
\begin{gathered}
\Pi(\rho, z)=\frac{2}{\varkappa_{1}^{2}}\left[\frac{\partial^{2}}{\partial z^{2}} \frac{\exp \left(-\varkappa_{1} R\right)}{R}\right. \\
\left.-\left(\frac{\partial^{3}}{\partial z^{3}}-\varkappa_{1}^{2} \frac{\partial}{\partial z}\right) I_{0}\left(r_{+}\right) K_{0}\left(r_{-}\right)\right], \\
P(\rho, z)=2 \frac{\partial}{\partial z} I_{0}\left(r_{+}\right) K_{0}\left(r_{-}\right), \\
S(\rho, z)=-\frac{2}{\varkappa_{1}^{2}} \frac{\partial}{\partial z} \frac{\exp \left(-\varkappa_{1} R\right)}{R} .
\end{gathered}
$$

Для определения поля линейной антенны необходимо проинтегрировать выражение (12) по длине антенны, заменив $\rho=\sqrt{x^{2}+y^{2}}$ на $\rho_{\eta}=\sqrt{(x-\eta)^{2}+y^{2}}$, $J \Delta_{x} \rightarrow J d \eta, \frac{\partial}{\partial x} \rightarrow-\frac{\partial}{\partial \eta}$

Обозначим $\left.\boldsymbol{E}^{(1)}\right|_{\varkappa_{0}=0}=\boldsymbol{\varepsilon}$ и учтем, что

$$
\frac{\partial}{\partial x}=\frac{\partial \rho_{\eta}}{\partial x} \frac{\partial}{\partial \rho_{\eta}}=\frac{x-\eta}{\rho_{\eta}} \frac{\partial}{\partial \rho_{\eta}}
$$

a

$$
\frac{\partial}{\partial y}=\frac{\partial \rho_{\eta}}{\partial y} \frac{\partial}{\partial \rho_{\eta}}=\frac{y}{\rho_{\eta}} \frac{\partial}{\partial \rho_{\eta}}
$$

тогда в результате интегрирования по $\eta$ получим

$$
\begin{gathered}
\mathcal{E}_{x}=\frac{i \omega \mu_{0}}{4 \pi} \\
\times J\left[\int_{-L}^{L} \Pi\left(\rho_{\eta}, z\right) d \eta+\left.\frac{x-\eta}{\chi_{1}^{2} \rho_{\eta}} \frac{\partial}{\partial \rho_{\eta}} P\left(\rho_{\eta}, z\right)\right|_{\eta=-L} ^{\eta=L}\right], \\
\mathcal{E}_{y}=\left.\frac{i \omega \mu_{0}}{4 \pi \varkappa_{1}^{2}} J \frac{y}{\rho_{\eta}} \frac{\partial}{\partial \rho_{\eta}} P\left(\rho_{\eta}, z\right)\right|_{\eta=-L} ^{\eta=L}, \\
\mathcal{E}_{z}=-\left.\frac{i \omega \mu_{0}}{4 \pi} J S\left(\rho_{\eta}, z\right)\right|_{\eta=-L} ^{\eta=L} .
\end{gathered}
$$

Выражения (19) - формальное решение задачи. В нем используются преобразования хорошо изученных модифицированных функций Бесселя. Для придания физической наглядности полученному решению сделаем еще ряд дополнительных шагов.

Начнем с определения поля на границе раздела $z=0$. Для этого необходимо знать П $(\rho, 0)$ и

$$
\left.\frac{1}{\rho} \frac{\partial}{\partial \rho} P(\rho, z)\right|_{z=0} \text {. }
$$




\section{Рассмотрим}

$$
\frac{1}{\rho} \frac{\partial}{\partial \rho} P(\rho, z)=\frac{2}{\rho} \frac{\partial}{\partial \rho} \frac{\partial}{\partial z} I_{0}\left(r_{+}\right) K_{0}\left(r_{-}\right) .
$$

Для предельного перехода к $z=0$ целесообразно продифференцировать по $\rho$ и $z$ произведение модифицированных функций Бессселя. Последовательно дифференцируя по $\rho$ и $z$, получим

$$
\begin{aligned}
\frac{1}{\rho} \frac{\partial}{\partial \rho} P(\rho, z) & =2\left[-\frac{r_{+}}{R^{3}} K_{0}\left(r_{-}\right) I_{1}\left(r_{+}\right)-\frac{r_{-}}{R^{3}} I_{0}\left(r_{+}\right) K_{1}\left(r_{-}\right)\right. \\
& \left.+\frac{\varkappa_{1}^{2} z}{2 R^{2}}\left(I_{0}\left(r_{+}\right) K_{0}\left(r_{-}\right)-I_{1}\left(r_{+}\right) K_{1}\left(r_{-}\right)\right)\right] .
\end{aligned}
$$

Отсюда следует, что при $z=0$

$$
\begin{aligned}
& \left.\frac{1}{\rho} \frac{\partial}{\partial \rho} P(\rho, z)\right|_{z=0} \\
& =-\frac{\varkappa_{1}}{\rho^{2}}\left[K_{0}\left(\frac{\varkappa_{1}}{2} \rho\right) I_{1}\left(\frac{\varkappa_{1}}{2} \rho\right)+I_{0}\left(\frac{\varkappa_{1}}{2} \rho\right) K_{1}\left(\frac{\varkappa_{1}}{2} \rho\right)\right] .
\end{aligned}
$$

В [9] показано, что выражение, находящееся в квадратных скобках, равно $2 /\left(\varkappa_{1} \rho\right)$, и соответственно имеем

$$
\left.\frac{1}{\rho} \frac{\partial}{\partial \rho} P(\rho, z)\right|_{z=0}=-\frac{2}{\rho^{3}} .
$$

Следовательно, на границе раздела снизу составляющая $\mathcal{E}_{y}$ электромагнитного поля будет равна

$$
\left.\varepsilon_{y}\right|_{z=0}=-\left.\frac{i \omega \mu_{0}}{2 \pi \varkappa_{1}^{2}} \frac{y}{\rho_{\eta}^{3}}\right|_{\eta=-L} ^{\eta=L}
$$

Применяя вышеописанную схему, после ряда несложных, но громоздких преобразований можно получить значение $\Pi(\rho, 0)$ :

$$
\Pi(\rho, 0)=\frac{2}{\varkappa_{1}^{2} \rho^{3}}\left[1-\left(1+\varkappa_{1} \rho\right) \exp \left(-\varkappa_{1} \rho\right)\right]
$$

и соответственно выражение для $\left.\varepsilon_{x}\right|_{z=0}$ :

$$
\begin{aligned}
\left.\mathcal{E}_{x}\right|_{z=0}= & \frac{i \omega \mu_{0}}{2 \pi \varkappa_{1}^{2}} J\left[\int_{-L}^{L} \frac{1-\left(1+\varkappa_{1} \rho_{\eta}\right) \exp \left(-\varkappa_{1} \rho_{\eta}\right)}{\rho_{\eta}^{3}} d \eta\right. \\
& \left.-\left.\frac{x-\eta}{\rho_{\eta}^{3}}\right|_{\eta=-L} ^{\eta=L}\right] .
\end{aligned}
$$

Анализируя (21) и (22), видим, что составляющая $\mathcal{E}_{x}$ в отличии от $\varepsilon_{y}$ содержит экспоненциальное слагаемое, связанное с волной, распространяющейся в нижней среде. Сравнение выражений (21) и (22) с результатами, полученными для поля на границе раздела при подходе к ней сверху [6], показывает их идентичность. Этого и следовало ожидать в силу непрерывности тангенциальных составляющих электрического поля на границе раздела двух сред.

Проанализируем поведение поля на некотором удалении от антенны и границы раздела. Учитывая, что компонента $\varepsilon_{z}$ имеет простую структуру и практически определяется волной, идущей от источника в точку приема, рассмотрим более подробно составляющую $\varepsilon_{y}$.

Считаем выполненным условие $\left|r_{+}\right| \gg 1$ или

$$
\left|(R+z) \frac{\varkappa_{1}}{2}\right| \gg 1 \text {. }
$$

Так как $z \leq 0$, то и

$$
\left|r_{+}\right|=\left|(R-z) \frac{\varkappa_{1}}{2}\right| \gg 1 .
$$

При выполнении условий $\left|r_{+}\right| \gg 1,\left|r_{-}\right| \gg 1$ можно воспользоваться асимптотическими разложениями для модифицированных функций Бесселя и следующих из них асимптотических представлений для произведений модифицированных функций Бесселя:

$$
\begin{aligned}
I_{0}\left(r_{+}\right) K_{0}\left(r_{-}\right) \sim \frac{\exp \left(\varkappa_{1} z\right)}{\varkappa_{1} \rho}\left[1-\frac{\varkappa_{1} z}{2 \varkappa_{1}^{2} \rho^{2}}\right], \\
I_{0}\left(r_{+}\right) K_{1}\left(r_{-}\right) \sim \frac{\exp \left(\varkappa_{1} z\right)}{\varkappa_{1} \rho}\left[1+\frac{\varkappa_{1} z}{2 \varkappa_{1}^{2} \rho^{2}}+\frac{\varkappa_{1} R}{\varkappa_{1}^{2} \rho^{2}}\right], \\
I_{1}\left(r_{+}\right) K_{0}\left(r_{-}\right) \sim \frac{\exp \left(\varkappa_{1} z\right)}{\varkappa_{1} \rho}\left[1+\frac{\varkappa_{1} z}{2 \varkappa_{1}^{2} \rho^{2}}-\frac{\varkappa_{1} R}{\varkappa_{1}^{2} \rho^{2}}\right], \\
I_{1}\left(r_{+}\right) K_{1}\left(r_{-}\right) \sim \frac{\exp \left(\varkappa_{1} z\right)}{\varkappa_{1} \rho}\left[1+\frac{3}{2} \cdot \frac{\varkappa_{1} z}{\varkappa_{1}^{2} \rho^{2}}\right] .
\end{aligned}
$$

Подстановка разложений (24) в (20) и получившегося результата в (19) дает

$$
\varepsilon_{y}=-\frac{i \omega \mu_{0}}{2 \pi \varkappa_{1}^{2}} \frac{y}{\rho_{\eta}^{3}}\left[1-\frac{z}{2 R_{\eta}}\left(1+\frac{3 z^{2}}{\rho_{\eta}^{2}}\right) \frac{1}{\varkappa_{1} R_{\eta}}\right] \exp \left(\varkappa_{1} z\right),
$$

где $R_{\eta}=\sqrt{\rho_{\eta}^{2}+z^{2}}$. Учтем, что $z / R_{\eta} \leq 1$ и на больших расстояниях, значительно превышающих скинслой, можно пренебречь членами, имеющими порядок $\left|l /\left(\varkappa_{1} R_{\eta}\right)\right|$, тогда из (25) получаем следующий результат:

$$
\left.\varepsilon_{y} \simeq \varepsilon_{y}\right|_{z=0} \exp \left(\varkappa_{1} z\right)
$$

Таким образом, в отличие от составляющей $\varepsilon_{z}$, которая описывается волной, распространяющейся исключительно в нижней среде, имеем волну, которая сперва распространяется в верхней среде без поглощения, а затем проникает на глубину вниз, испытывая изменения по экспоненциальному закону.

Выполнив аналогичные преобразования для $\mathcal{E}_{x}$, можно увидеть более сложную структуру формирования $\mathcal{E}_{x}$, которая будет суперпозицией двух волн, одна из которых, как и при формировании $\varepsilon_{z}$, определяется распространением исключительно в нижней среде, а другая распространяется в верхней среде, впоследствии проникая вниз.

Учитывая, что величина прямого поля в нижней среде есть величина порядка отброшенных членов в асимптотическом разложении (24), им можно пренебречь. Поэтому закономерность изменения $\varepsilon_{x}$ с глубиной будет подобна $\mathcal{E}_{y}$. 


\section{Заключение}

Таким образом, в результате проведенных вычислений получены выражения для компонент электрического поля, возбуждаемого линейной заводненной антенной, в виде интегралов, содержащих быстроосциллирующие функции. В области частот, для которых выполняется условие малости волнового числа, в вакууме по сравнению с модулем волнового числа в нижней среде, что справедливо для морской воды для частот от нуля до нескольких килогерц, найдено решение задачи в виде хорошо изученных специальных функций.

Показано, что вертикальная компонента поля формируется волной, распространяющейся исключительно в нижней среде. При этом на границе раздела горизонтальная компонента электрического поля, перпендикулярная к антенне, определяется полем, распространяющимся в верхней среде, а продольная - суммой волн в нижней и в верхней среде. В то же время при удалении от антенны изменение составляющих $\mathcal{E}_{x}$ и $\varepsilon_{y}$ с глубиной происходит по экспоненциальному закону.

\section{Список литературы}

[I] Sommerfeld A. // Ann. Phys. 1909. Vol. 28. P. 665-736

[2] Weil H. // Ann. Phys. 1919. Vol. 60. P. 481-500.

[3] Бурсиан B.P. // Теория электромагнитных полей, применяемых в электроразведке. Л.: Недра, 1972. 367 с.

[4] Wait J.R. // Electromagnetic waves in stratified madia. NY: Pergamon press, 1962. $372 \mathrm{p}$.

[5] Макаров Г.И., Новиков В.В., Рыбачек С.Т. // Распространение электромагнитных волн над земной поверхностью. М.: Наука, 1991. 196 с.

[6] Вешев А.В. // Электропрофилирование на постоянном и переменном токе. 2-е изд. перераб. и дополн. Л.: Недра, 1980. 391 c.

[7] King R.W.P., Owens M., Wu Tai Tsun. Lateral Electromagnetic Waves. Theory and Applications to Communications, Geophysical Exploration and Remote Sensing. NY: Springer-Verlag, 2011. 746 p.

[8] Жданов М.C. // Теория обратных задач и регуляция в геофизике. М.: Научный Мир, 2007. 661 с.

[9] Градштейн И.С., Рыжик И.М. // Таблицы интегралов, сумм, рядов и произведений. М.: Физматгиз, 1962. 1100 с.

[10] Терещенко П.Е. // ЖТФ. 2010. Т. 80. Вып. 7. С. 147-150.

[II] Куваркин А.Б., Новикова Е.И. // Вычислительная математика и математическая физика. 1981. Т. 21. № 5. C. 1091-1099. 